\title{
التباين الكمي لإمدادات المياه عبر الشبكة العامة للمياه ببلدية مصراتة
}

$$
\begin{aligned}
& \text { (2018.2012): الأسباب والبدائل } \\
& \text { أبوبكر علي سليمان الصول } 1 \\
& \text { كلية الآداب - جامعة مصراتة }
\end{aligned}
$$

$$
\begin{aligned}
& \text { تاريخ التقديم: 04-05-2019 ، تاريخ القبول: 12-09- 2019، نشر إلكترونيا في 15-09-2019 } \\
& \text { https://doi.org/10.36602/faj.2019.n14.02 }
\end{aligned}
$$

\section{ملخص البحث:}

يستعرض هذا البحث مصادر المياه التي تغذي الشبكة العامة للمياه الحضرية، وتقصي

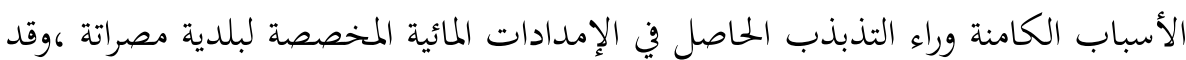
تمحورت الدراسة حول إعطاء نبذة عامة عن مصادر التزود بالمياه في السابق، وما لحق بها من عطل، وحلول البديل المتمثل في النهر الصناعي، وعلى الرغم من المخصصات الجيدة للمنطقة من هذا المصدر، إلا أنه لا يعتمد عليه على المدى البعيد؛ بسبب التذبذبات التي تحصل لإمداداته بين الفينة والأخرى، والتي كانت مثار بحث في هذه الدراسة، كما خلصت الدراسة إلى جملة من النتائج منها: توقف كلي لمصادر التغذية القديمة، فضلا عن عدم كفاية المياه المخصصة من منظومة النهر الصناعي، وتعرضها للتذبذب من حين لآخر على مدى الفترة السابقة، وبذلك ثم اقتراح بعض من التوصيات التي يرى الباحث الفائدة منها

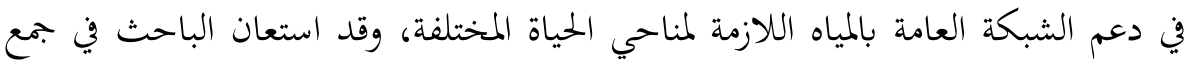
البيانات ببعض المراجع العلمية و التقارير الصادرة عن المؤسسات ذات العلاقة، فضلا عن المقابلات الشخصية مع المدراء والمسؤولين بالإدارات المختلفة وفق المتاح.

الكلمات المفتاحية: المياه الحضرية ـ الإمداد المائي ـ حقول الآبار الجوفية ـ الشبكة العامة لمياه ـ البدائل المطروحة.

a.assol@art.misuratau.edu.ly ${ }^{1}$ 


\section{Quantitative Contrast of Urban Water Supplies Through the Public Network of Water in Misurata Municipality (2012- 2018):Reasons and Alternatives.}

\section{Abubaker Ali Alssol}

Misurata University

\section{Abstract:}

This paper reviews the water resources that supply the urban public water network, and investigates the reasons beyond the fluctuation in water supplies that assigned to Misurata Municipality. The paper also focuses on giving an overview of the sources of water in the past, the damage caused to it as well as the alternative solution which is the man-made river. Despite the good allocations to the region from this source, it is not reliable in the long term, due to fluctuation that occur to its supplies from time to time, which was the subject of research in this study. In terms of data collection, the researcher collected the data from the references and official reports issued by relevant institutions, furthermore, making interviews with managers and officials in various administrations in the water sector. The study concluded the following results: total cessation of old supplying sources, the insufficient water that came from the man-made river system and exposed to fluctuate from time to time in the previous period. Thus, some of the recommendations have been suggested, that the researcher finds them useful in supporting the water public network that might be needed for different aspects of life.

Key words: Urban water, water supply, ground wells fields, the public network of water, proposed alternatives.

$$
\begin{aligned}
& \text { 1. المقدمة: } \\
& \text { الماء هو عصب الحياة، كونه مصدرا أساسيا للشرب وتجهيز الغذاء، كذلك يعدّ } \\
& \text { عماد الاستخدامات الحضرية والصناعية والزراعية وبعض الخدمات كأعمال البناء والعمران، } \\
& \text { وبذلك تكمن أهميته، فبتوفره تستمر مناحي الحياة، وبفقده تتوقف، و يمثل الامداد المائي }
\end{aligned}
$$


الحضري أولوية مطلقة لجهات الاختصاص بالدولة في توفير المياه ،وذلك بوضع الخطط والدراسات وتنفيذ المشاريع ذات العلاقة بهذا المصدر، والتي تكفل استدامة توفر المياه ذات الاستخدام الحضري وضمان وصولها لمستخدميها كما ونوعا. يتم التركيز في هذا البحث على إمدادات المياه عبر الشبكة العامة للمياه، فالملاحظ أن هذا المورد يعاني من التذبذب في الإمدادات عبر شبكة المياه الحضرية ببلدية مصراتة، في ظل تزايد الطلب على المياه مع محدودية مصادر التزويد، لذا يأتي البحث كمحاولة لكشف الغموض حول تذبذب المياه الحضرية عبر الشبكة العامة ببلدية مصراتة وتحري الأسباب وراء ذلك، فضلا عن معرفة البدائل التي توفر هذا المورد في حالة الانقطاع من المصدر. : 1.1

ما هي المصادر المائية التي تزود الشبكة العامة للمياه في بلدية مصراتة؟

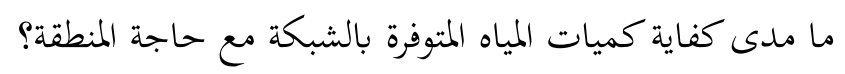
هل يمكن أن تكون الأسباب الفنية هي المسئولة عن تذبذب الإمداد المائي بالشبكة؟ همبه إلى أي مدى يمكن أن تسهم الأسباب الخارجة عن امكانيات شركة المياه في حدوث

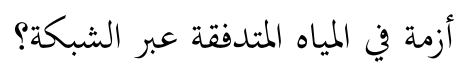

ما هي البدائل المطروحة لاستمرار تدفق المياه الحضرية عبر الشبكة العامة للمياه؟

\section{2 . 2 فرضيات البحث:}

محدودية مصادر التزويد ـ النهر الصناعي ـ أسهم في عملية تذبذب الإمدادات المائية. هناك علاقة بين شدة الطلب وعدم إيفاء الإمدادات المائية بحاجة السكان. اتساع دائرة الشبكة وتقادمها أسهم في حدوث عمليات فقدان لكميات المياه المحدودة. توجد عمليات تخريب للشبكة العامة قد تكون متعمدة في ظل الضعف الأمني العام. 
لا توجد بدائل للإمداد المائي في حالة توقف المصدر الرئيسي، أو تكون غير كافية لتوفير الكميات المطلوبة. 1 3 ـ أهداف البحث يهدف البحث إلى تحقيق الآتي:

تصنيف مصادر المياه المتعددة، وأولوياتا في تزويد الشبكة العامة للمياه. تحديد الأسباب الكامنة وراء تذبذب إمدادات المياه عبر الشبكة العامة للمياه

$$
\text { الحضرية. }
$$

تحديد المصادر البديلة المتاحة والمقترحة لحل مشكلة تذبذب المياه بالشبكة.

\section{1}

يلقي الضوء على مواطن الخلل الذي أسهم في عمليات تذبذب إمدادات المياه عبر الشبكة العامة للمياه الحضرية.

التعبف بمجهودات جهات الاختصاص لحلحلة العجز القائم في هذا المورد. يسهم في نشر الوعي بأهمية المياه في حياة المواطنين ببلدية مصراتة، وترشيد استهلاكها

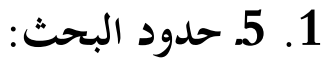

المجال المكاني : ويتمثل في الحدود الجغرافية لبلدية مصراتة حسب التقسيم الإداري لسنة (1986) خريطة (1)، حيث البحر المتوسط من الشمال والشمال الشرقي وبلدية زليتن من الغرب وبلدية بني وليد من الجنوب الغربي وبلدية سرت من الجنوب الشرقي، أما

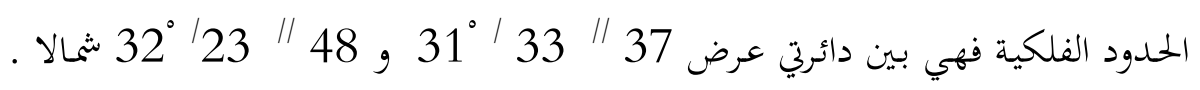

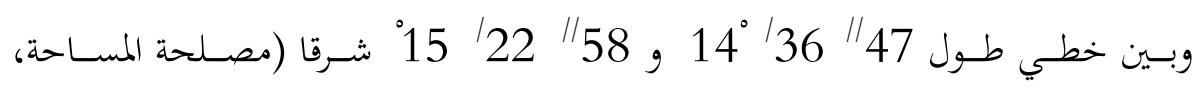
(33) 1978 
مجلة كلية الآداب- العدد 14، ديسمبر، 2019 التباين الكمي لإمدادات المياه ببلدية مصراتة

المجال الزماني: تشمل الدراسة الفترة الواقعة بين سنتي (2012 ـ 2018)

خريطة(1)موقع منطقة الدراسة

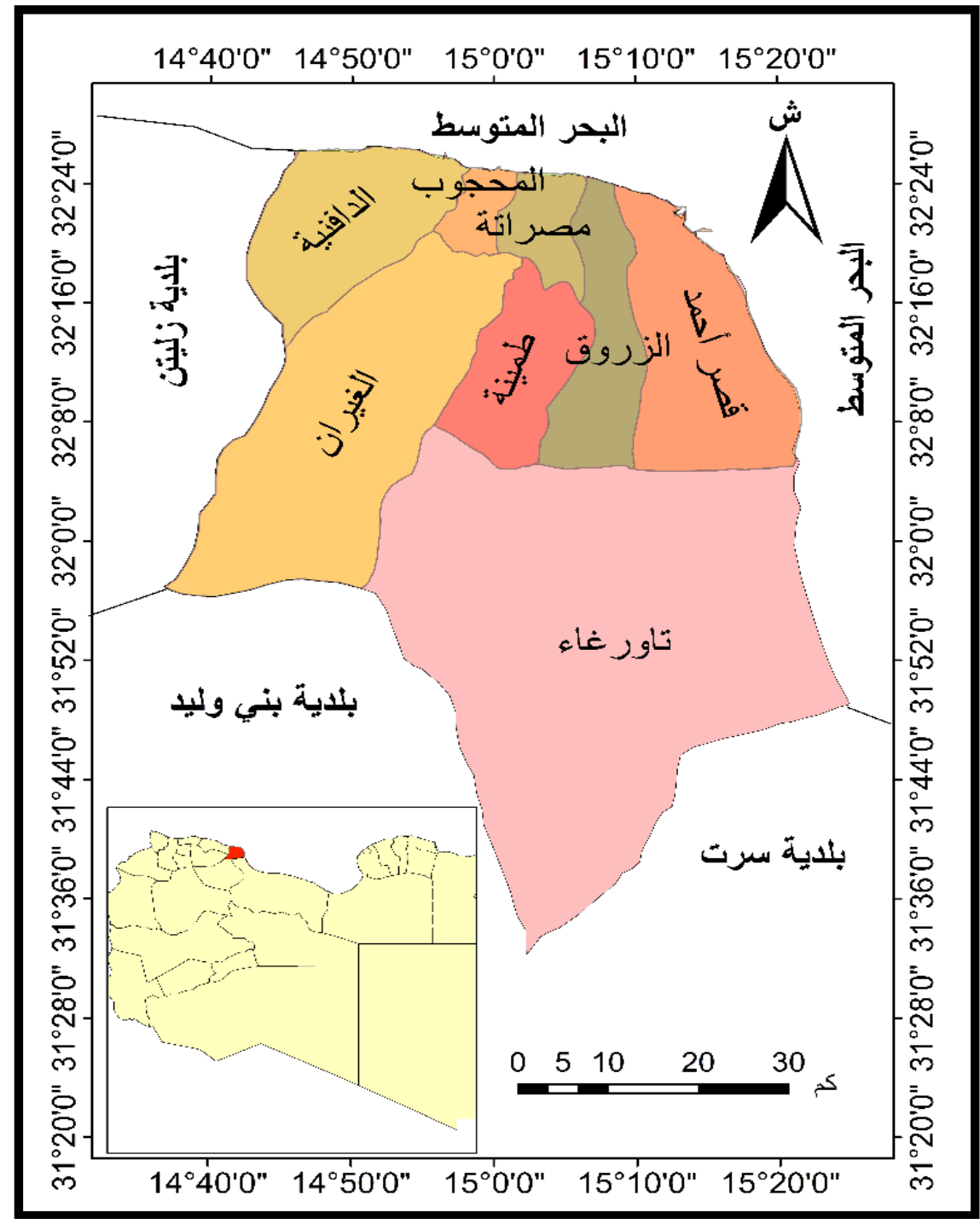

المصدر:الباحث باستخدام برنامج Arc Gis، استنادا الى اللجنة الشعبية للمرافق ببلديةخليج

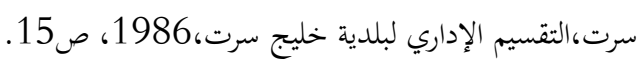




\section{1}

استخدم الباحث المنهج الوصفي التحليلي في وصف الظاهرة محل البحث معتمدا على البيانات المتوفرة التي أسهمت في استخلاص عدد من النتائج متبوعة بجملة من التوصيات. 2. مصادر التزويد بالثبكة العامة للمياه الحضرية:

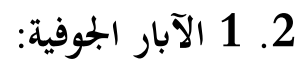

كانت منطقة الدراسة تعتمد على مياه الآبار الجوفية بحقول السكت وفلاجة وطمينة والمحجوب وتاورغاء في الفترة من (1970 ـ 1999م) في تغطية احتياجاتا من المياه

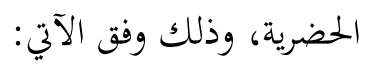
أ ـ حقل آبار السكت: يقع جنوب غرب مدينة مصراتة بحوالي (12) كم، يتكون من (27) بئراً حفرت في الفترة (1970 ـ 1975م) انخفض عدد الآبار المنتجة على مر السنين حتى وصل حاليا إلى بئر واحد عامل.

ب ـ حقل آبار طمينة: يقع جنوب غرب مدينة مصراتة بحوالي (15) كم يتكون من (22) بئرا حفرت مابين (1972 ـ 1973م) وبإنتاجية (10000) متر مكعب/اليوم للحقل، انخفضت إلى أن وصلت إلى (8) أمتار مكعبة/اليوم لبئر واحد عامل فقط. ج • حقل آبار فلاجة: يقع جنوب غرب مدينة مصراتة بحوالي (17) كم يحتوي على (24) بئرا حفرت مابين عامي (1980 ـ 1982) بإنتاجية تقدر (8000) متر مكعب/اليوم، انخفضت إلى أن وصلت إلى عدد (3) آبار عاملة بإنتاجية حوالي (10) أمتار مكعبة/اليوم • خريطة (2). 
خريطة (2) التوزيع الجغرافي لحقول المياه بمصراتة.

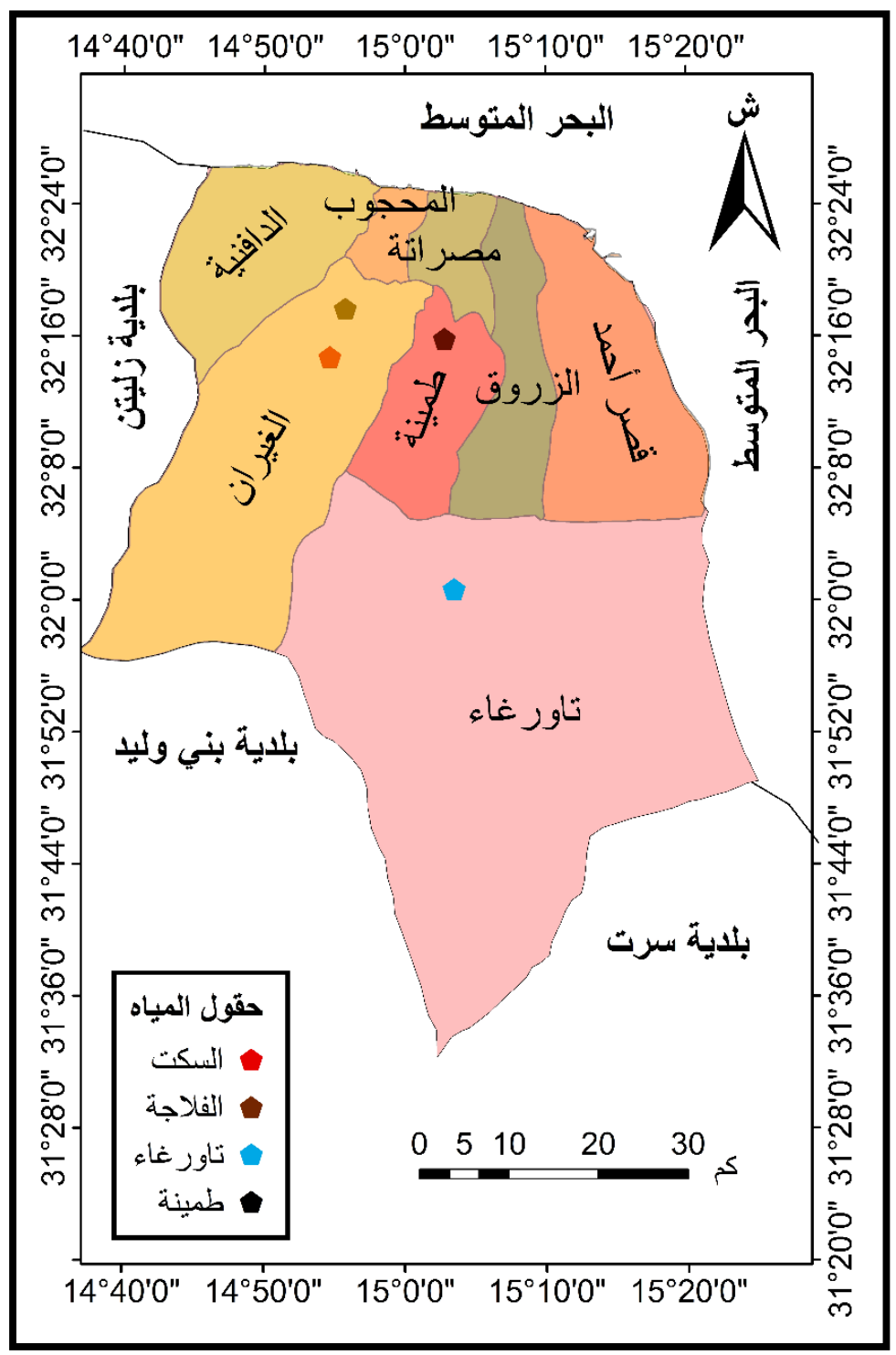

المصدر : الباحث باستخدام برنامج Arc Gis استنادا الى جهاز تحديد المواقع (GPS)

د . حقل آبار زاوية المحجوب: يقع غرب المدينة ويضم عدد (5) آبار حفرت عام

(1971م) بإنتاجية وصلت إلى (1000) متر مكعب/اليوم وكانت مياهها غير جيدة؛ 
لارتفاع نسبة الملوحة (4000) ملجرام/اللتر أدى إلى إغلاقها. كما يوجد عدد (3) آبار بمنطقة أبوروية غير صالحة للشرب منها بئر واحدة يستغل من قبل أصحاب سيارات نقل المياه. (الشركة العامة للمياه والصرف الصحي، إدارة التشغيل والصيانة بالمنطقة الوسطى، 2014م، ص2 ـ ـ 4).

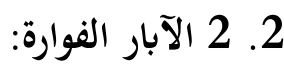

حفرت بجموعة من الآبار الفوارة، بغرض دعم الشبكة العامة للمياه، قبل وصول مياه النهر الصناعي، في الفترة التي بدأت تعجز فيها آبار الحقول سالفة الذكر عن توفير الحد الأددن من متطلبات المياه الحضرية بالشبكة وتتمثل في: أ. بئر عبدالرؤوف: يقع جنوب غرب المدينة بحوالي (25) كم، حفر سنة (1987م) بإنتاجية حوالي (300) متر مكعب/الساعة، وتم ربطه مباشرة بخزان حقل فلاجة، ومن ثم إلى الشبكة العامة، وبالرغم من أن البئر بحالة جيدة، إلا أنه توقف عن الضخ نتيجة التعديات التي حدثت على الكوابل الكهربائية المغذية له. ب ـ بئر السكت: يقع على بعد (200) متر من خزان بتميع مياه حقل السكت، حفر سنة (1982م) لزيادة كمية الإمداد المائي بمحطة السكت الرئيسية بإنتاجية (50) متراً

\section{مكعباً /الساعة.}

ج · آبار تاورغاء: تشمل (8) آبار عميقة تم حفرها مابين سنتي (1984 ـ 1987م) تبعد مسافة (45) كم جنوب شرق مدينة مصراتة بإنتاجية (3500 ـ 5000) متر مكعب/الساعة وهي مياه عسرة لذا تم إنشاء محطة معالجة على هذه الآبار بطاقة تصميمية (60000)متر مكعب/اليوم بدأت العمل سنة (1997) يتم ضخ المياه المعالجة إلى مركز 
توزيع المياه بالسكت بإنتاجية (25000 ـ 30000) متر مكعب/اليوم في السنوات الأولى، ثم تدرجت في الانخفاض، ثم توقفت سنة (2011م) لتعرضها للتدمير. (الشركة العامة للمياه والصرف الصحي، إدارة التشغيل والصيانة بالمنطقة الوسطى،2014م،

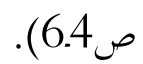

\section{3 2 3 2}

تم إنشاء محطات تحلية المياه أسهمت بنسب متفاوتة في دعم الشبكة العامة وشملت: آ ـ محطة تحلية كرزاز: تقع جنوب شرق مركز المدينة وتعتمد على عدد (4) آبار عميقة ارتوازية مالحة بإنتاجية تقدر مابين (3000 ـ 4000) متر مكعب/اليوم للبئر الواحد، وتتم التحلية بطريقة التناضح العكسي وقد اشتغلت المحطة في الفترة مابين عامي (1986 ـ 1991م) بإنتاجية تقدر بـ (9500) متر مكعب/اليوم، بمياه ذات جودة عالية لتختلط بمياه آبار محطة طمينة عالية الملوحة، وتسهم في دعم الشبكة العامة للمياه الحضرية، وقد توقفت عن العمل منذ (1991م)، لعدم توفر قطع الغيار وارتفاع تكاليف مواد التشغيل.( بيت المال و بيت المال، 2007،صن3233). ب . محطة تحلية الحديد والصلب: تتبع الشركة الليبية للحديد والصلب، تشتغل بنظام التبخير الوميضي"، بطاقة تصميمية (30000) متر مكعب/اليوم ، أسهمت في دعم الشبكة العامة بالفترة (1988 ـ 1997م) لتغذية الجزء الشرقي للمدينة بحوالي (15000)متر مكعب/اليوم، توقفت المحطة حاليا عن تغذية الشبكة العامة للمياه. (الشركة العامة للمياه والصرف الصحي، إدارة التشغيل والصيانة بالمنطقة الوسطى، .) (15) 2014

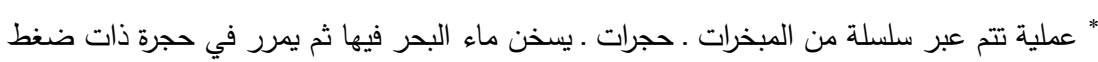

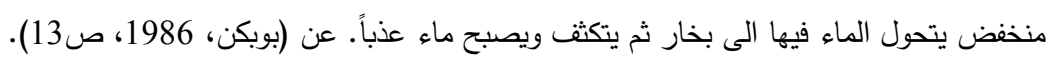


تعدّ المصادر سالفة الذكر متوقفة عن تغذية الشبكة العامة بسبب مجموعة من المعوقات نذكر منها:

1 ـ السحب الجائر من قبل المواطنين أصحاب المزارع الذين قاموا بحفر آبار بنفس الحوض الذي يغذي آبار حقول المياه الخاصة بالشبكة العامة، مما ترتب عنه انخفاض منسوب المياه بمعدل عالٍ وجفاف الخزان السطحي، مع زحف مياه البحر للمحافظة على التوازن الهيدروليكي، سبب في تملح المياه الجوفية وتدهور نوعيتها بشكل واضح. 2 ـ انتهاء العمر الافتراضي للمعدات ووسائل استخراج ونقل وتحميع وضخ المياه في حقول المياه وبعض محطات التحلية والمعالجة. 3 ـ استيلاء بعض المواطنين على بعض آبار حقول المياه، خاصة في حقلي السكت وفلاجة. 4 ـ تعرض بعض الآبار للسلب، سواء معدات التشغيل أو كوابل الكهرباء المغذية لها، في الفترة قبل (2012م).( الصول، 2015م، ص213 ـ 216).

2. 4 مياه النهر الصناعي

تعتمد منطقة الدراسة حاليا بشكل رئيسي في التغذية بمياه الشرب والاستخدامات الحضرية على مياه النهر الصناعي المنقولة من حوض مرزق وجبل الحساونة عبر المسار الشرقي للمرحلة الثانية للنهر الصناعي، ويعدّ من أكبر الأحواض المائية الجوفية في العالم حيث تبلغ مساحته حوالي 450 ألف كم² بطبقة صخرية حاوية للماء بسمك 800 متر وسعة تخزينية تقدر بجوالي 4800 كم مكعب ، كما يتميز بنوعية مياه جيدة جدا صالحة لجميع الاستخدامات حيث تقدر نسبة الأملاح الذائبة الكلية بحوالي 300 جزء في المليون

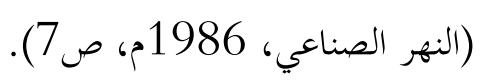


تم ربط مياه النهر بخزاني توزيع المياه الرئيسيين بمنطقة السكت سعة (25000) متر مكعب/اليوم لكل خزان، وبدأت تغذية المنطقة بمياه النهر الصناعي سنة (1999م) بحوالي (25000) متر مكعب/اليوم وصلت في عام (2015م) إلى (90000متر مكعب/اليوم، حيث تمثل هذه الكمية ما يزيد عن (95)\% من إجمالي الإمداد المائي لبلدية مصراتة. (الشركة العامة للمياه والصرف الصحي، إدارة التشغيل والصيانة بالمنطقة الوسطى، 2014،ص2).

على الرغم من أن هذه الكمية المخصصة لاتصل كاملة إلى مستحقيها؛ بسبب الفاقد المقدر بـ (20\%) نتيجة التسربات الحاصلة في الشبكة العامة للمياه (الشركة العامة للمياه والصرف الصحي، إدارة التشغيل والصيانة بالمنطقة الوسطى، 2014،ص16) فهي تعدّ أقل من حاجة السكان بمنطقة الدراسة والتي تقدر بـ (126823)متراً مكعباً /اليوم(") وفقا لتقديرات عدد السكان لسنة 2018م والبالغ (377450) نسمة،(مصلحة الأحوال المدنية، فرع المنطقة الوسطى، قسم المعلومات والتوثيق، بيانات غير منشورة)، هذا عدا السكان المقيمين بشكل مؤقت، وعلى الرغم من ذلك فإن هذه الكميات تتعرض للتخفيض والانقطاع من وقت لآخر، مما يترتب عليه زيادة الطلب على هذا المصدر، ويجعل منه سلعة يتم احتكارها من قبل أصحاب سيارات نقل المياه، ويلاحظ من خلال الجدول (1) الذي يوضح كميات المياه المتدفقة بالشبكة العامة للمياه ببلدية مصراتة خلال الفترة (2012 ـ 2017م) بالمتر المكعب شهريا، تذبذبا في مخصصات المنطقة من هذا المصدر الأمر الذي يدعو الى التقصي والبحث في معرفة الأسباب. 3. - - الأسباب والبدائل

(") وفق معدل استهلاك الفرد من المياه بواقع 336 لتر/اليوم. عن (كعيبة ،2007،ص355). 


\section{1 أسباب الأزمة المائية خلال الفترة (2018.2012م).}

يعدّ انقطاع الإمداد المائي عبر منظومة النهر الصناعي بين الحين والآخر، هو السبب الرئيسي للأزمة المائية بمنطقة الدراسة، على اعتبار أها المصدر الوحيد للمياه الحضرية، فضلا عن ضعف الصيانة والعمرة لكل المرافق الخدمية للشبكة العامة للمياه ؛ نتيجة لضعف التمويل المالي ـ (اسماعيل جهان، مدير مكتب التشغيل والصيانة لشبكات المياه/ إدارة الوسطى، مقابلة بتاريخ 2018/11/22م) وما يترتب عنها من فقدان لكميات من مياه الشبكة بالتسرب كذلك غياب الإمدادات البديلة عن مياه النهر والناتج عن توقف مصادر المياه القديمة سالفة الذكر. يمكن إجمال أسباب الانقطاع في:

أ. أسباب أمنية: حيث تعرضت بعض آليات استخراج المياه من الآبار للسرقة والتخريب، وشملت محولات الكهرباء، وتدمير كامل لمعدات حجرة التشغيل، وسرقة كوابل الكهرباء والقياس، مما سبب في توقف حوالي (88) بئرا حتى هاية أكتوبر 2018م وفقدان ما يقارب من (350) ألف متر مكعب من المياه خلال ذلك الشهر ـ في شهر أكتوبر 2018م توقف عدد (12) بئرا دفعة واحدة ـ كذلك تزايد حالات التعدي على مسارات خطوط أنابيب نقل مياه النهر بالتوصيلات غير القانونية، لغرض الحصول على المياه للاستخدام الحضري والزراعي، وهذه التوصيلات تشكل أضرارا على المعدات والأنبوب الرئيسي. كذلك حالات الاعتداء والخطف التي طالت العديد من المشغلين لمنظومة الحساونة (فنيين ومهندسين) من قبل مجموعات مسلحة خارجة عن القانون، بغرض تعطيل عمل المنظومة، وهذا من شأنه الحد من قدرة فرق التشغيل والصيانة والمراقبة من القيام بأعمالهم المنوطة . هم. (جهاز تنفيذ وإدارة مشروع النهر الصناعي (2،1، 2018 
ب ـ أسباب فنية: حالات عطل فني في بعض معدات التشغيل ،كعل أحد صمامات خزان التجميع بالشويرف في الفترة من شهر 2017/10 إلى شهر 2018/5م محا ترتب عنه الخفاض عملية الضخ في المسار الشرقي للمنظومة، التي تتغذى منها منطقة الدراسة إلى مابين 30 ـ 40\% فضلا عن تأخر استيراد قطع الغيار البديلة، نتيجة الإجراءات الإدارية والمالية المتعلقة بالدولة، بعد أن تعرضت مخازن قطع الغيار للسرقة في الفترة ما بين (2013 2017)، كذلك أدت حالات الفصل التام في الشبكة العامة للكهرباء(BLACK OUT) إلى توقف تشغيل المنظومة بين الحين والآخر، التي يحتاج إعادة تشغيلها وتدفق المياه من جديد إلى مابين (1. 3) أيام، وذلك لمراعاة توازن الضغط بالأنابيب، والذي هو من اختصاص المهندسين المشرفين بقسم التحكم والمعلومات بالمنظومة، فضلا عن توقف منظومة السدادة القرضابية بعد سنة 2011م التي تربط بين منظومة النهر بالفرع الشرقي (السرير. تازربو. بنغازي) ومنظومة النهر بالفرع الغربي (الحساونة ـ سهل الجفارة) التي تحف الى سد العجز الذي قد يطرأ في إحدى المنظومتين من الأخرى(محمد القاعد، رئيس قسم التحكم والمعلومات، منظومة الحساونة .سهل الجفارة، مقابلة شخصية بتاريخ (2019/3/13 
جدول (1) كميات المياه المتدفقة بالشبكة العامة للمياه ببلدية مصراتة خلال الفترة (2012 ـ 2017م) بالمتر المكعب شهريا.

\begin{tabular}{|c|c|c|c|c|c|c|c|c|c|c|c|c|}
\hline\{ & 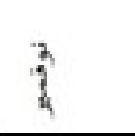 & $\sqrt{3}$ & 1 & 3 & $-\frac{3}{2}$ & th & $\frac{3}{x}$ & 3 & $\frac{3}{3}$ & $\frac{2}{x}$ & يائر & \\
\hline 2160793 & 2901090 & 2160793 & 2091090 & 2160793 & 2146533 & 2092590 & 2091090 & 2077290 & 2197683 & 2077590 & 2077590 & 2012 \\
\hline - & 2245680 & 2265780 & 2565780 & 2813560 & 2813560 & 2813560 & 2813560 & 2813560 & 2813560 & 2541280 & 2723560 & 2013 \\
\hline 2715680 & 2715680 & 2715680 & 2715680 & 2715680 & 2715680 & 2715680 & 2715680 & 2715680 & 2245680 & 2245680 & 2245680 & 2014 \\
\hline 2439300 & 2439240 & 2437300 & 2439280 & 1135641 & 2565680 & 2415680 & 2715680 & 2355680 & 2446800 & 2625680 & 2715680 & 2015 \\
\hline 1761000 & 2705000 & 2702000 & 2279100 & 3019500 & 3320000 & 2626000 & 376000 & 1814010 & 1213900 & 2439240 & 2439240 & 2016 \\
\hline 1863150 & 53000 & 503000 & 1512500 & 2257000 & 209900 & 2419050 & 1508000 & 1958500 & 1184000 & 2232120 & 2259500 & 2017 \\
\hline
\end{tabular}

المصدر : الشركة العامة للمياه والصرف الصحي، إدارة التشغيل والصيانة بالمنطقة الوسطى، 2018م، (غير منشور). 
مجلة كلية الآداب- العدد 14، ديسمبر، 2019 التباين الكمي لإمدادات المياه ببلدية مصراتة

\section{2 البدائل المطروحة من قبل شركة المياه:}

نتيجة اعتماد منطقة الدراسة على مصدر واحد، وهو مياه النهر الصناعي، وما لحق بهذا المصدر من أضرار في الفترة التي شملتها الدراسة، فضلا عن بعض المشكلات التي قد تواجه مشروع النهر الصناعي لاحقا كارتفاع تكلفة الضخ نتيجة هبوط منسوب المياه الجوفية في مناطق السحب (الجديدي، 2008م، ص46)، أصبحت الحالة تستدعي توفير مصادر أخرى بديلة متمثلة في مجموعة المقترحات التالية:

1.2.3اقترحت إدارة المنطقة الوسطى بالشركة العامة للمياه والصرف الصحي، إنشاء محطة تحلية لمياه البحر بإنتاجية (300000) متر مكعب/اليوم، وتم إحالة المقترح إلى دوائر القرار بالدولة الليبية، وصدر القرار رقم (295) لسنة 2013م من مجلس الوزراء بإنشاء محطة تحلية سعة (85000) متر مكعب/اليوم ولم يدخل المشروع حيز التنفيذ بعد. (إسماعيل علي جهان، مدير مكتب التشغيل والصيانة بالشركة العامة للمياه/ إدارة الوسطى، مقابلة شخصية بتاريخ 2018/11/20).

2.2 .3 اقترح حفر عدد (106) آبار جوفية بمحطات توزيع المياه ـ الخزانات العلوية . بالمنطقة بمتوسط عمق (300 ـ 350) مترا لتعويض النقص أو انقطاع المياه عبر منظومة النهر الصناعي، وتخصيص محطة تنقية ومعالجة مياه لكل موقع من المواقع المقترحة لحفر هذه

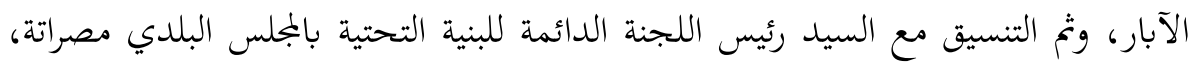

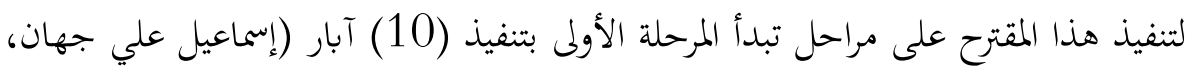

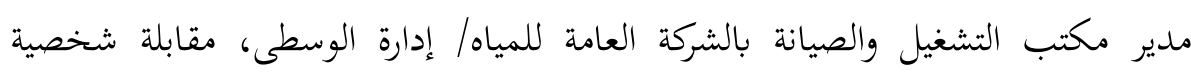
بتاريخ 2018/11/20) كما هو موضح بالجدول (2) والشكل (1) مائل وفي مقابلة مع السيد رئيس اللجنة الدائمة للبنية التحتية بالمجلس البلدي مصراتة، صرح بأن التنفيذ سيقتصر على حفر (5 أو 6) آبار في المرحلة الأولى، وذلك بسبب 
ارتفاع أسعار و تكاليف الحفر والتجهيز لهذه الآبار، وسيتم التنفيذ حسب المقترح المرسل من إدارة التشغيل والصيانة بالمنطقة الوسطى بالشركة العامة للمياه والصرف الصحي، وتم مخاطبة الهيئة العامة للمياه بالمنطقة الوسطى لتتولى الإشراف والمتابعة، واختيار الشركة المنفذة لعملية الحفر وفق المواصفات الفنية، ومن المتوقع أن يبدأ التنفيذ خلال (10) أيام من تاريخ المقابلة الشخصية (علي أبوستة، رئيس اللجنة الدائمة للبنية التحتية بالمجلس البلدي مصراتة، مقابلة شخصية 2018/12/9م). جدول(2) مواقع الآبار المقترحة من شركة المياه.

\begin{tabular}{|c|c|c|c|c|c|}
\hline \multirow[t]{2}{*}{ عدد الآبار } & \multicolumn{2}{|c|}{ الإحداثيات } & \multirow[t]{2}{*}{ الموقع } & \multirow[t]{2}{*}{ المركز } & \multirow[t]{2}{*}{ ت } \\
\hline & شمالا & شرقا & & & \\
\hline 1 & 322203.37 & 151055.60 & خزان الملايطة & شرق المدينة & 1 \\
\hline 1 & 322300.65 & 145730.40 & خزان سعدون & غرب المدينة & 2 \\
\hline 1 & 322155.50 & 145920.00 & خزان الكرامة & غرب المدينة & 3 \\
\hline 1 & 322301.99 & 150640.63 & خزان يدر القديم & وسط المدينة & 4 \\
\hline 1 & 322109.51 & 150525.54 & خزان 9 يوليو & وسط المدينة & 5 \\
\hline 1 & 322356.12 & 150444.72 & خزان باشاغا & وسط المدينة & 6 \\
\hline 1 & 322136.38 & 150435.72 & مستشفى الطوارئ & وسط المدينة & 7 \\
\hline 1 & 322216.90 & 150308.27 & خزان راس فريدغ & وسط المدينة & 8 \\
\hline 2 & 321939.70 & 150600.00 & محطة تحلية كرزاز & طمينة & 9 \\
\hline 10 & & & & & \\
\hline
\end{tabular}

المصدر: الشركة العامة للمياه والصرف الصحي، إدارة التشغيل والصيانة بلمنطقة الوسطى، 2018م.

إن عملية حفر الآبار من قبل المواطنين أصبحت ظاهرة تثير الانتباه بالرغم من الحاجة الماسة للماء التي اضطرتم لاتخاذ مثل هذه التدابير حيث تتم العملية في العموم 
بشكل عشوائي دون الرجوع لجهات الاختصاص مما قد يترتب عليه بعض الآثار السلبية

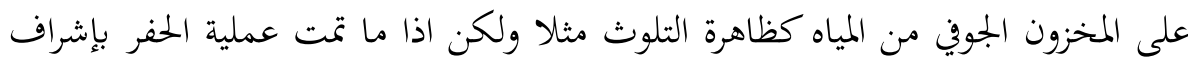
مؤسسات مختصة وكان تصميم البئر وفق دراسات علمية فسوف يساعد ذلك على تأمين

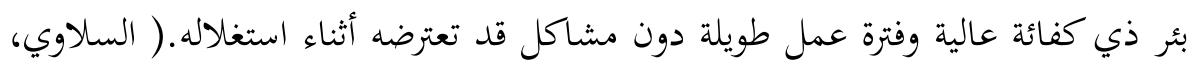

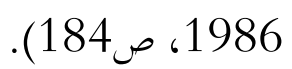

كما تقوم الشركة من خلال مكتب خدماها، بتقديم خدمة توصيل المياه إلى المواطنين الذين لاتصل إليهم المياه عبر الشبكة العامة للمياه، وذلك عن طريق سيارات نقل المياه ـ عبوة (10) أمتار مكعبة ـ رغم أن هذه الخدمة دون المستوى المطلوب؛ بسبب عدم كفاية عدد السيارات، حيث تمتلك الشركة عدد (20) سيارة على مستوى بلدية مصراتة، منها (4) عاطلة، موزعة على ستة مراكز لتوزيع المياه هي (9يوليو، قصر أحمد، الغيران، زاوية المحجوب، الجزيرة، طمينة) بواقع (2 ـ 4) سيارات لكل مركز توزيع، وتقدر الرحلات اليومية لكل سيارة (5) رحلات، حيث تعتبر مياه النهر الواصلة إلى مراكز التوزيع عبر الشبكة العامة للمياه، هي مصدر التزويد لهذه السيارات. (عبدالعزيز ارفيدة، رئيس قسم الخدمات المساندة، بمكتب خدمات شركة المياه والصرف الصحي ببلدية مصراتة، مقابلة شخصية 2018/11/22). 


تجلة كلية الآداب-

شكل (1) التوزيع المكاني للآبار المقترحة ببلدية مصراتة

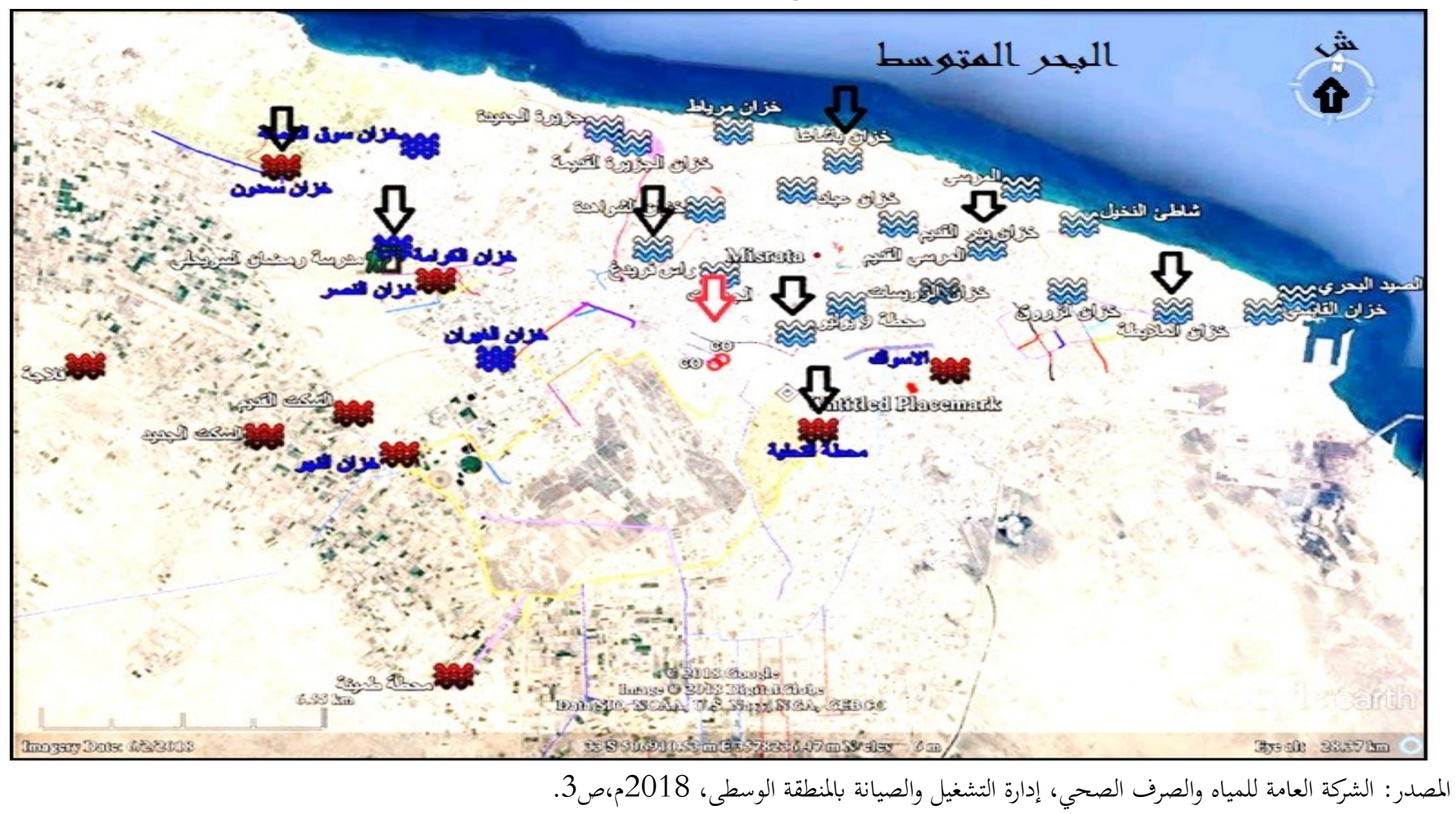




\section{3 أعمال الإنشاء والصيانة للشبكة خلال الفترة (2013 ـ 2018):}

تعدّ الشبكة العامة للمياه في بعض أجزائها متهالكة، توجد بها انسدادات وتسرب،

نتيجة كثرة الكسور، وهذا يرجع في معظمه إلى قدم إنشاء الشبكة العامة، حيث بحاوز عمرها (30) سنة.نتج عنه ارتفاع نسبة الفاقد من مياه الشبكة، بالرغم من محاولات الشركة المستمرة في تنفيذ الصيانات، وبحديد لبعض الخطوط القديمة، كذلك تنفيذ خطوط جديدة لمناطق سكنية لم تصل لها الشبكة من قبل. فمنذ سنة (2013م) تم تنفيذ عدد من خطوط الشبكة الرئيسية والفرعية في عدة نواحٍ من منطقة الدراسة بلغت أطوالها حوالي (12053) متراً في خطوط الشبكة الرئيسية، وحوالي (39123) متر في خطوط الشبكة الفرعية تتراوح أقطارها (160) ملم في الشبكات الرئيسية و (32 ـ 110) ملم في الشبكات الفرعية، من المواسير ذات نوع بولي ايثلين. (الشركة العامة للمياه والصرف الصحي، 2014،

(إدارة الطلب:

في ظل الموارد المائية المحدودة التي تشهدها الدولة الليبية عامة، ومنطقة الدراسة خاصة، أسهمت عوامل أخرى في تذبذب امدادات المياه عبر الشبكة الأمر الذي يتطلب إدارة فعالة وصارمة تتصرف في حدود كميات المياه المتاحة، وفق أولويات تحكمها قواعد واضحة لتحقيق الطلب المتزايد على المياه، وهو ما يعرف بإدارة الطلب (الطلحي،ب ت، ص2322، (321،). ومنها:

وجود نسبة من الإسراف والتبذير عند بعض المستهلكين في استعمالات المياه لمختلف الأغراض، كرش الشارع وري الحدائق وغسيل السيارات، كذلك إِهمال عمليات الصيانة 
الدورية لبعض المرافق الصحية بالمنازل والمحال الحندمية وهنا يتطلب تفعيل مكاتب الجباية

$$
\text { لترشيد الاستهلاك. }
$$

•انخفاض أعداد فرق الصيانة على مستوى المنطقة ترتب عنه تأخر مواجهة التسربات المتعددة في خطوط الشبكة العامة التي تبقى لفترة تسبب في ضياع كميات من المخصصات المائية.

•ترتيب أولويات استهلاك المياه عبر الشبكة بداية للاستخدام المنتلي والخدمي ثم للأغراض الخدمية الأخرى، أو تبني استخدام الأنابيب الثنائية ـ كما هو متبع في بعض الأقطار العربية كدولة الكويت ـ حيث أنابيب تنقل المياه العذبة لاستغلالها في الأغراض المنزلية وأخرى تخصص للمياه غير العذبة (قليلة الملوحة) للأغراض الخدمية

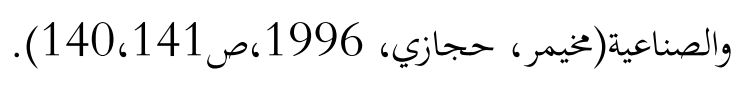
4. النتائج والثوصيات:

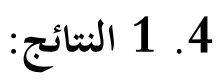
يمكن استخلاص النتائج الآتية: - م 1. توقف تزويد إمدادات المياه للشبكة من حقول الآبار الجوفية بالمنطقة بشكل عام قبل سنة (2012م). 2 ـ من الممكن إعادة استغلال بعض آبار بعض الحقول للمساهمة في إمدادات المياه. 3. تعرض بعض الآبار العاملة لعمليات فب وسرقة مما أذى إلى توقفها عن تغذية الشبكة. 4. تعدّ مياه النهر الصناعي هي المصدر الوحيد لإمدادات المياه بالشبكة العامة للمياه الحضرية للمنطقة. 
مجلة كلية الآداب- العدد 14، ديسمبر، 2019 التباين الكمي لإمدادات المياه ببلدية مصراتة

5. تعرض منظومة النهر الصناعي لعمليات توقيف لإمدادات المياه لأكثر من مرة خلال الفترة (2018.2013).

6. انتهاء العمر الافتراضي لجزء من الشبكة العامة للمياه أسهم في فقدان كميات من المياه. 7. لا توجد بدائل واقعية لمصدر الإمداد الحالي ـ النهر الصناعي ـ للشبكة العامة للمياه الحضرية بمنطقة الدراسة.

4. 2 . 2 التوصيات:

1. البدء في تنفيذ محطة معالجة وتحلية مياه البحر كبديل استراتيجي عن مياه النهر الصناعي.

2ـ البدء في تنفيذ مقترح شركة المياه بحفر آبار بمحطات توزيع المياه المنتشرة بالمنطقة. 3. العمل على صيانة بعض الآبار القديمة بحقول المياه بالمنطقة. 4ـ وضع خطط استراتيجية وتنفيذها مرحليا تمدف المى بجديد جميع مرافق الشبكة العامة للمياه بالمنطقة. 5ـ زيادة عدد سيارات نقل المياه لعدم كفاية المتاح منها، وتوفير قطع الغيار لصيانة العاطل منها.

\section{قائمة المراجع: - مائ}

بوبكن، روي (1986). تحلية مياه البحر. بيروت: دار الآفاق الجديدة. بيت المال، سليم محمد و بيت المال، عمر محمد (2007). دراسة الوضع المائي في منطقة

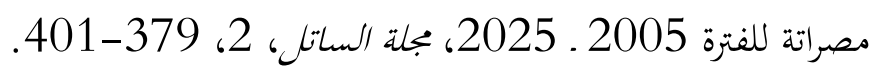

الجديدي، حسن محمد (2008). البدائل المطروحة لمواجهة تناقص المياه الجوفية. الزاوية: دار شموع الثقافة. 
مجلة كلية الآداب- العدد 14، ديسمبر، 2019 التباين الكمي لإمدادات المياه ببلدية مصراتة

جهاز تنفيذ وإدارة مشروع النهر الصناعي (1986) النهر الصناعي. المؤلف.

جهاز تنفيذ وإدارة مشروع النهر الصناعي(2018). تقرير عن منظومة الحساونة ـ سهل الجنارة. المؤلف.

السلاوي، محمود سعيد (1986). المياه الجوفية بين النظرية والتطبيق. مصراتة: الدار الجماهيرية للنشر والتوزيع والاعلان.

الشركة العامة للمياه والصرف الصحي المنطقة الوسطى (2014). تقرير (غير منشور)عن الوضع المائي والصرف الصحي ببلدية مصراتة لسنة 2014.

الشركة العامة للمياه والصرف الصحي المنطقة الوسطى، (2018). تقرير (غير منشور)عن الوضع المائي والصرف الصحي ببلدية مصراتة لسنة 2018.

الصول، أبوبكر علي،(2015)، الموازنة المائية في منطقة مصراتة ـ ليبيا، (رسالة دكتوراه. غير منشورة ). كلية الآداب جامعة طنطا.

الطلحي، جاد الله عزوز، (ب ت ). حتى لا نموت عطشا،ط2.إدارة المطبوعات والنشر، اللجنة الشعبية العامة للثقافة والاعلام.

الكالوش، رمضان عبدالله ، (2018) مدير إدارة التشغيل والصيانة بالشركة العامة للمياه بالمنطقة

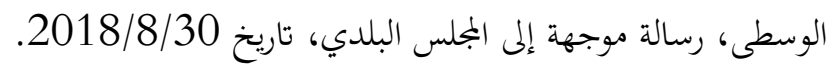

كعيبة، الحسين سالم (2007). ملدى انسجام الموارد المائية مع حجم السكان بمنطقة مصراتة. مجلة الساتل، 2، 351-359.351.

مخيمر، سامر و حجازي، خالد (1996). أزمة المياه في المنطقة العربية. سلسلة عالم المعرفة، المجلس الوطني للثقافة والفنون والآداب ، الكويت، مايو، العدد 209. مصلحة المساحة (1978) الأطلس الوطني. طرابلس: وزارة (أمانة) التخطيط. 